\title{
Mastopexia y prótesis. Revisión a los 5 años
}

\section{Mastopexy with and implant. A 5 year follow-up}

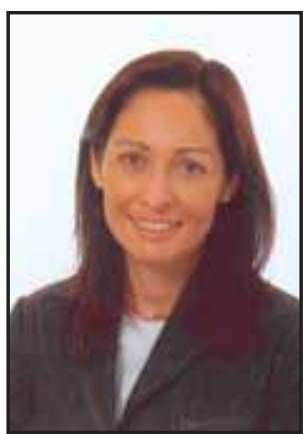

Moreno Gallent, I.

Moreno Gallent, I.*, Ribera Pons, M.**

Resumen

Se realiza una revisión de la técnica de Mastopexia y prótesis que realiza el autor. Una de las alteraciones más difíciles de resolver para el cirujano plástico es el de las mamas caídas o muy caídas que presentan un gran exceso de piel, y un escaso volumen de la glándula mamaria. Estos serían los casos adecuados para la realización de esta técnica. Es muy frecuente y se produce tras grandes pérdidas de peso, lactancias repetidas o prolongadas, o después de la involución que se produce en la mujer en el proceso de envejecimiento. Otra indicación para la realización de esta técnica se da en mamoplastias de aumento secundarias en las que hay piel sobrante y un grado de atrofia glandular producido por la compresión del implante durante mucho tiempo. Se presenta la técnica quirúrgica y se discuten las ventajas y complicaciones, así como la evolución en el tiempo. La técnica realizada presenta pocas complicaciones y un buen grado de satisfacción por parte de los pacientes.

Palabras clave Mastopexia. Implantes mamarios.

Código numérico 52103-52105; 52114
A revision of Mastopexy with an implant technique is carried out by the author. One of the most difficult disturbances that the surgeon faces is the ptotic breasts, which present a great skin excess and a low volume of mammary gland. These would be the adecuate cases for this technique. It is very frequent and it is produced after big weight losses, repeated or extended lactations, or after involution produced by the aging process. Another indication for carrying out this process would be secundary mammaplasty with skin remaining and atrophy due to the compression of the inmplant for a long time. The technique is presented, and the advantages and complications are discussed, as well as the evolution through time.

This technique has few complications and a high level of patient satisfaction.

Key words Mastopexy. Breast implant.

Código numérico 52103-52105; 52114 


\section{Introducción}

La ptosis mamaria corresponde a una alteración estética en la que se observa un descenso de la mama por debajo del pliegue inframamario. Son muchas las causas que se le atribuyen y resulta de difícil corrección para el cirujano. Muchas veces a la ptosis mamaria se une una hipotrofia, es decir un tamaño pequeño de la mama que va a dificultar la corrección y el logro de un volumen adecuado, si no es mediante la colocación de un implante mamario.

Son muchas las clasificaciones de las ptosis mamarias existentes en la literatura: Duformentel (1959)(1); Lalardrie (1974) (2); Regnault (1976) (3); Bostwick (1983) (4) y una de las más recientes Kirwan (2002) (5), que además establece la técnica quirúrgica para cada estadío.

En este trabajo se lleva a cabo una revisión de la técnica practicada por los autores, en la que se realiza Mastopexia con pedículo superior e interno y colocación de implantes de suero salino mamarios en posición retropectoral. La cicatriz resultante será periareolar y vertical por encima del pliegue inframamario.

Los objetivos que se pretenden a largo plazo son fundamentalmente el relleno del polo superior de la

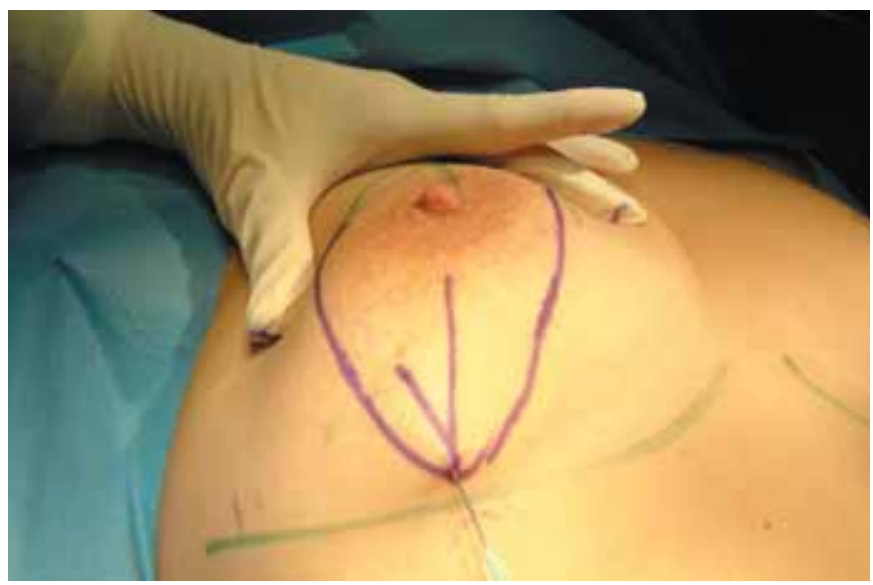

Fig. 1: Marcaje de las incisiones.

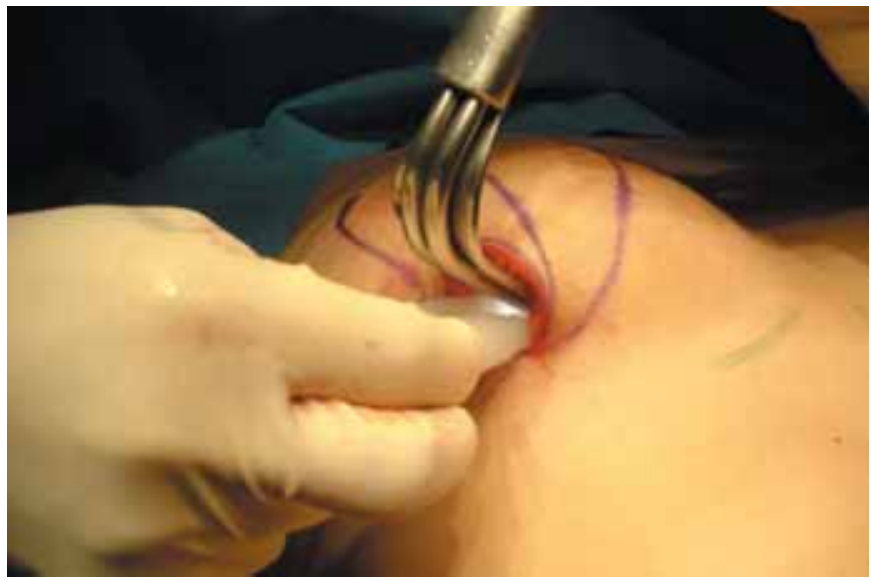

Fig. 3: Colocación del implante. mama y la persistencia del complejo areola-pezón en su posición a lo largo del tiempo.

\section{Material y Método}

Se hace una revisión de 150 casos intervenidos en los últimos cinco años, en los que se llevó a cabo la técnica que describimos. La edad de las pacientes osciló entre los 23 y los 65 años.

Previamente realizamos un marcaje de la paciente en bipedestación y utilizamos el patrón de Wise que nos sirve de referencia, aunque lo modificaremos tras colocar el implante. Trazamos líneas que determinan el pliegue inframamario, la altura de la areola y la futura posición a la que se colocará la nueva areola. Tras ello realizamos un marcaje de la incisión oblicua para lo colocación de los implantes en el polo inferior de la mama, a fin de preservar el colgajo de pedículo interno que vamos a realizar con posterioridad,y tras disecar la glándula y abordar el músculo pectoral, despegamos un plano subpectoral que servirá para alojar el implante.

Utilizamos prótesis de suero salino. Los tamaños que hemos utilizado han oscilado en estas pacientes entre $175 \mathrm{cc}$ y $225 \mathrm{cc}$, rellenando siempre con más 25 -



Fig. 2: Despegamiento plano retropectoral.

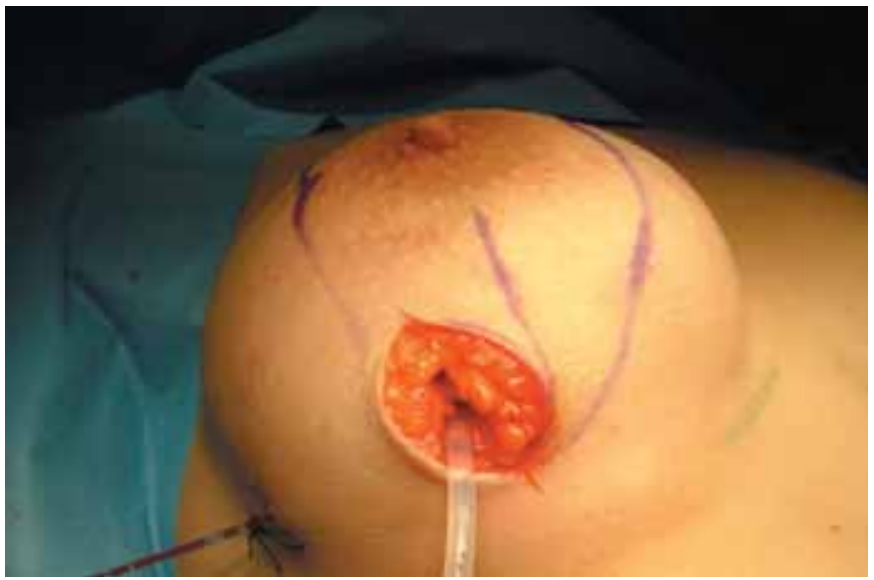

Fig. 4: Relleno del implante de suero salino. 


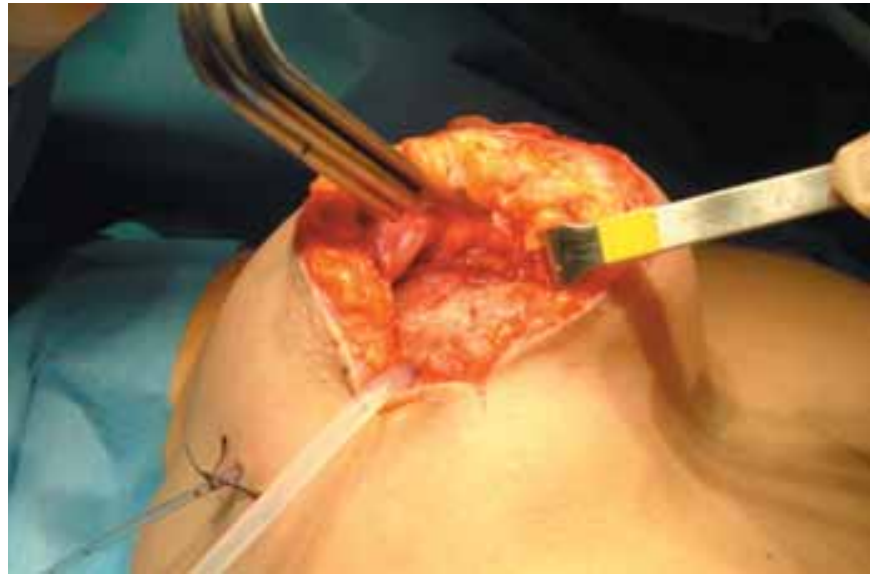

Fig. 5: Despegamiento del plano subgalndular.

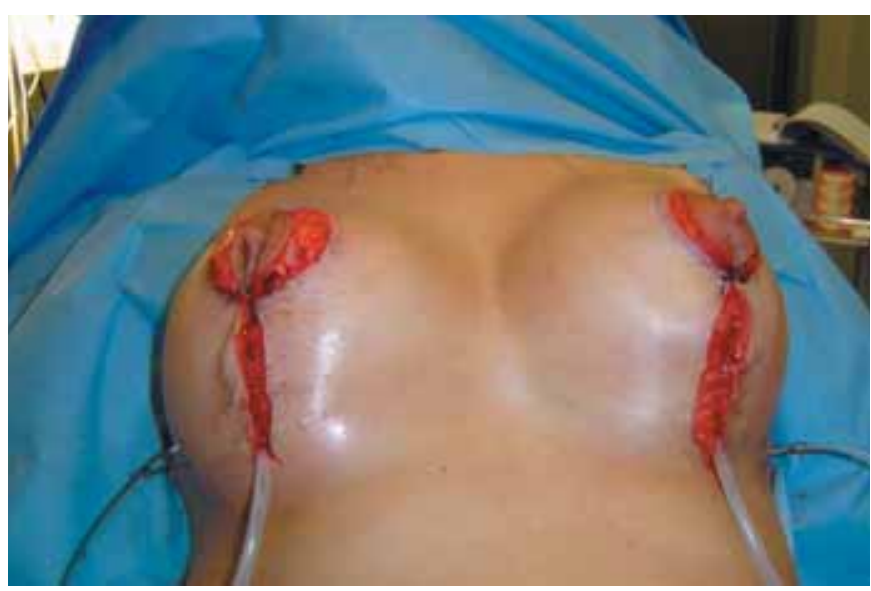

Fig. 7: Marcaje de las incisiones para finalizar la mastopexia.

35cc para evitar los pliegues y la posterior deflación de la prótesis. Colocamos el implante y lo llenamos de la forma explicada. Una vez conseguido el volumen que deseamos, procedemos a marcar la piel sobrante, y practicar la resección cutánea y subir el complejo areola-pezon a su nueva posición.

Una vez resecada la piel y tras desepidermizar el complejo areola-pezón, tallamos un colgajo de pedículo interno y superior que introducimos en la base del complejo areola-pezon y que fijamos con el fin de conseguir la protusión del mismo y el aspecto de mama cónica que pensamos es el adecuado. Tenemos de esta forma dos planos de despegamiento: uno retropectoral donde queda alojado el implante y otro retroglandular que permite el remodelado y posterior fijación de la glándula mamaria. Dejamos siempre vendaje compresivo y drenajes.

\section{Resultados}

Los resultados se muestran en las imágenes adjuntas (Fig. 1-12) donde se aprecia que el resultado que se pretende se mantiene con el tiempo; la evolución presentada es de cinco años.

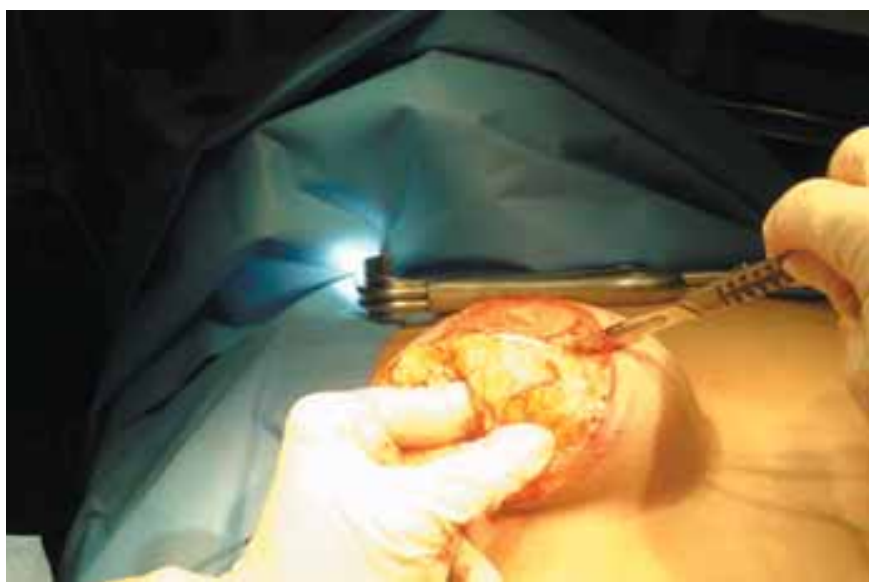

Fig. 6: Realización de un colgajo de pedículo interno y superior para proyectar el complejo areola-pezón.

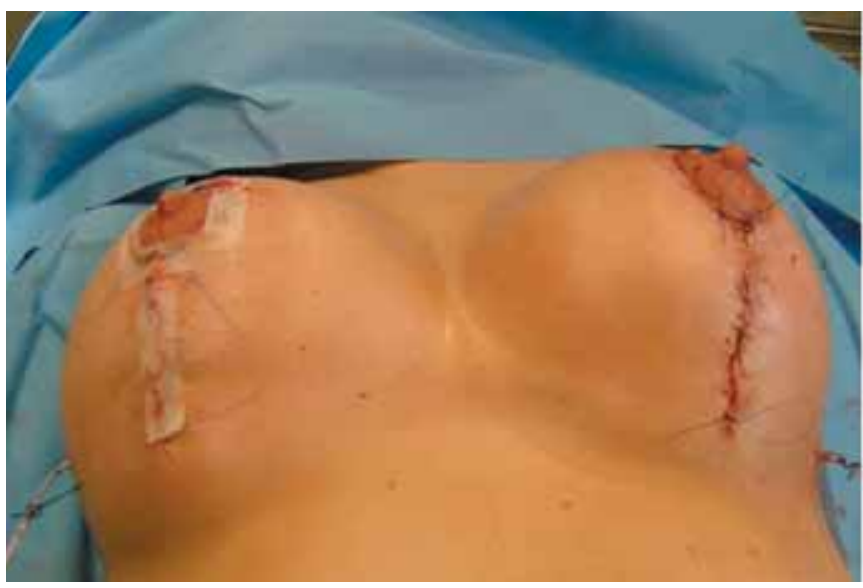

Fig. 8: Resultado preoperatorio inmediato.

Las ventajas de la técnica serían a nuestro juicio: el ser una técnica que deja poca cicatriz, que gracias a la utilización de implantes se mantiene intacto el relleno del polo superior, y finalmente el que dado el colgajo que introducimos en la base del complejo areolapezón se evita la recidiva de la ptosis en la mayoría de los casos.

Los inconvenientes serían el que se trata de una técnica que deja una cicatriz periareolar y otra vertical, que incluye la colocación de implantes que como todos sabemos son cuerpos extraños que presentan complicaciones (desplazamientos, roturas, contracturas...) y que además es una técnica sujeta a la improvisación puesto que hasta que no colocamos los implantes no podemos llevar a cabo un patrón preestablecido.

En cuanto a complicaciones podríamos citar las propias de la cirugía: infecciones, cicatrices hiperpigmentadas, pero citaremos las que nosotros hemos tenido con la técnica que presentamos. Tuvimos un desplazamiento de la prótesis, tras herniación de la misma hacia el polo superior de la mama que se solucionó cerrando el espacio periprotésico. Son relativamente frecuentes las asimetrías de una mama con respecto a la otra (10\% de los casos), pero en todos los 


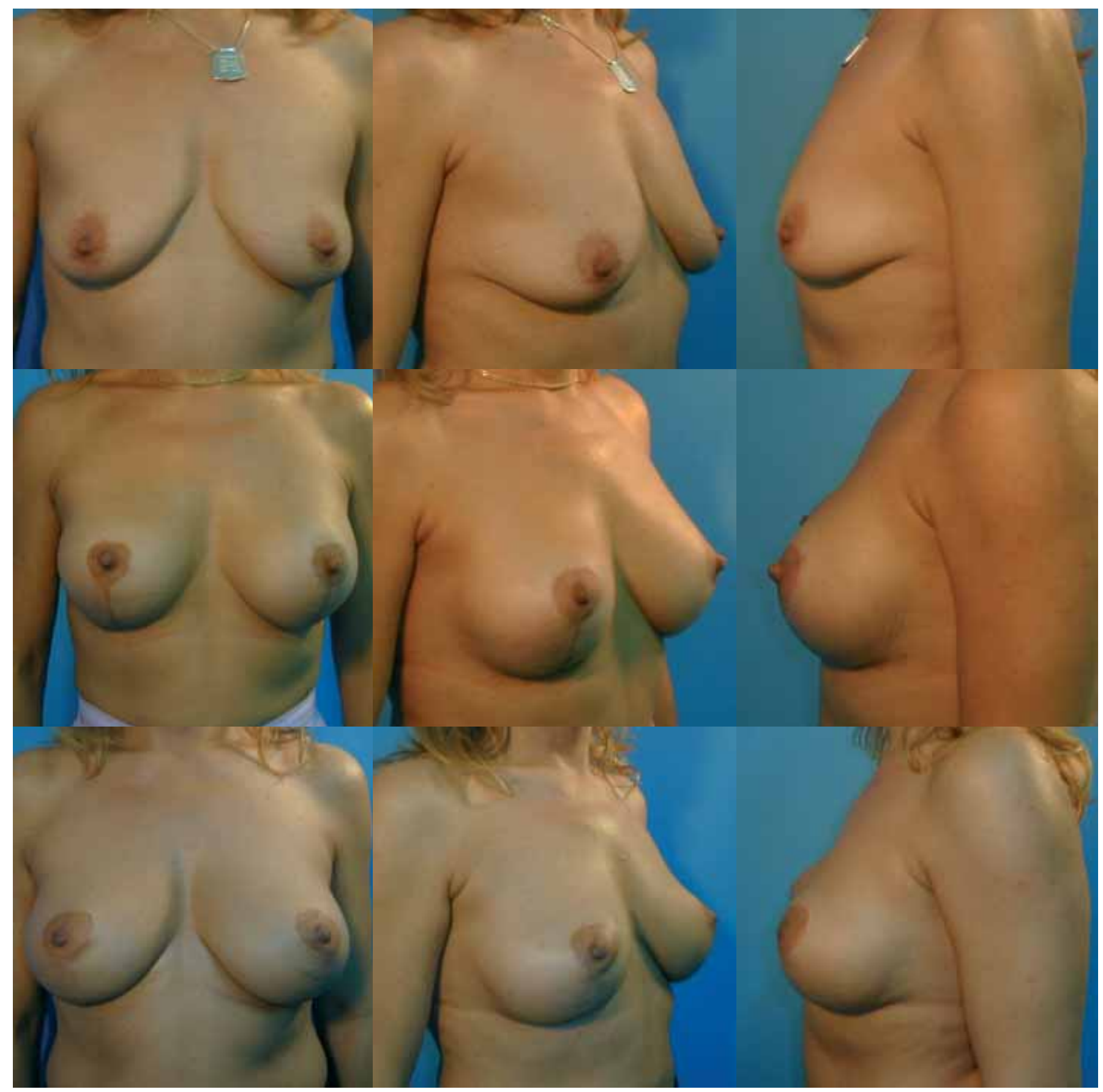

Fig. 9: Paciente 1. Mujer 50 años, Preoperatorio, postoperatorio 1 año y postoperatorio a los 5 años.

casos advertimos a la paciente y le recordamos que la simetría nunca se va a conseguir; son poco los casos que precisan de un retoque. Son muy infrecuentes las contracturas capsulares (hay que recordar que colocamos los implantes retropectorales y de suero salino). Advertimos a todas las pacientes que los implantes no son definitivos y que si sufren una deflación habrá que cambiarlos.

\section{Discusión}

El objetivo de toda cirugía en la mama es conseguir la forma más bonita, con el tamaño adecuado y con la mínima cicatriz. A lo largo del tiempo se han desarrollado múltiples técnicas que todos conocemos : en 1985, Puckett (6) describió técnicas periareolares para pequeñas elevaciones del complejo areola-pezón; en 1990 Regnault (7) utilizó pequeños implantes para incremen- 


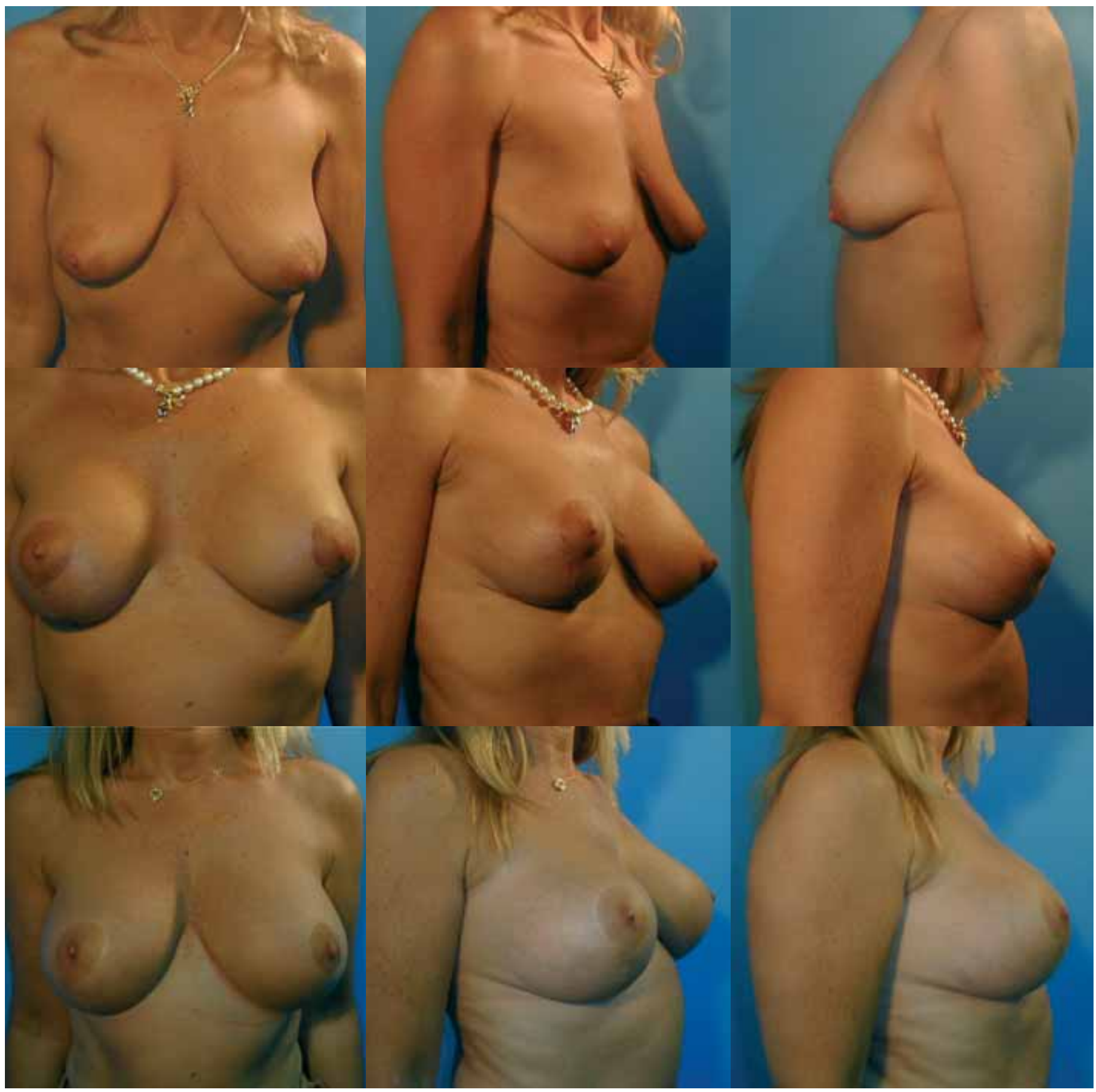

Fig. 10: Paciente 2. Mujer 47 años, Preoperatorio, postoperatorio 1 año y postoperatorio a los 5 años.

tar el volumen: Lejour (8) determinó las pautas de la mamoplastia vertical, pero no hay en la actualidad una técnica definitiva dada la dificultad que conlleva el problema de ptosis mamaria con hipotrofia asociada (9).

Utilizamos implantes de suero salinos porque nos dan buen resultado a largo plazo y pocas complicaciones (10). Nuestro índice de deflaciones a los 5 años es inferior al $1 \%$.

Consideramos que es importante colocar primero el implante para así asegurar el tamaño que queremos obtener y poder realizar la pexia con la mayor seguridad sin que luego se quede una mama a excesiva tensión, lo que nos permite obtener una mejor cicatriz resultante $(11,12)$. Si la mama queda a excesiva tensión la cicatriz se ensanchará.

El despegamiento de la mama en posición retroglandular permite un remodelado de la misma y la colocación del colgajo de pedículo interno en la base 


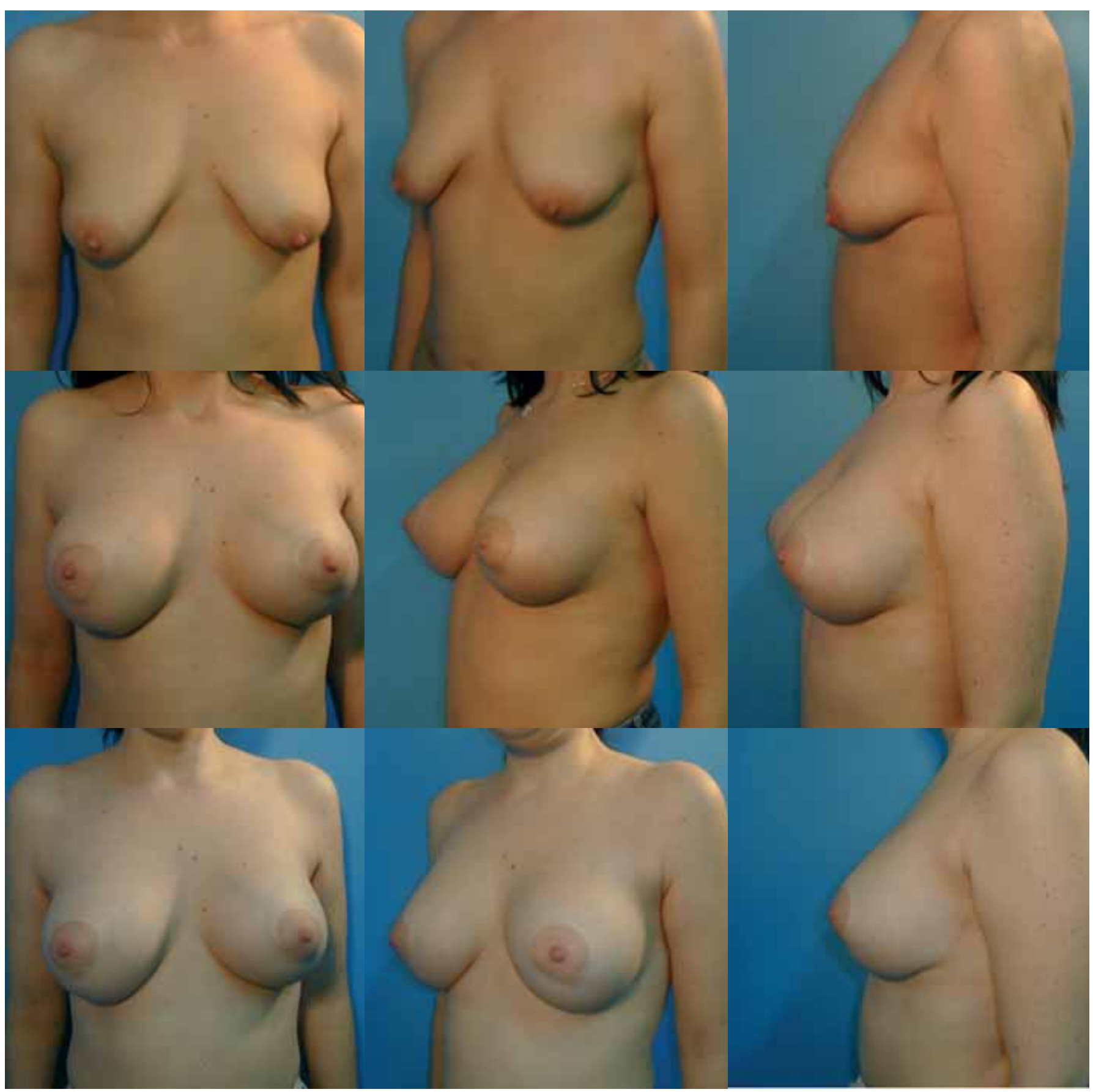

Fig. 11: Paciente 3. Mujer 25 años, Preoperatorio, postoperatorio 1 año y postoperatorio a los 5 años.

del complejo areola-pezón va a permitir la protusión del mismo y el dar la forma cónica que consideramos es la ideal de la mama.

\section{Conclusiones}

Como conclusiones, y teniendo en cuenta la casuística presentada, pensamos que la técnica que empleamos es adecuada para conseguir el objetivo pretendido que es el de una mama con volumen adecuado, escasa cicatriz y la permanencia a lo largo del tiempo del relleno del polo superior .

\section{Dirección del autor}

Dra. Isabel Moreno Gallent

Avda. de las Cortes Valencianas 28- B

46015 Valencia. España

e-mail: imoreno@clinicaisabelmoreno.com 


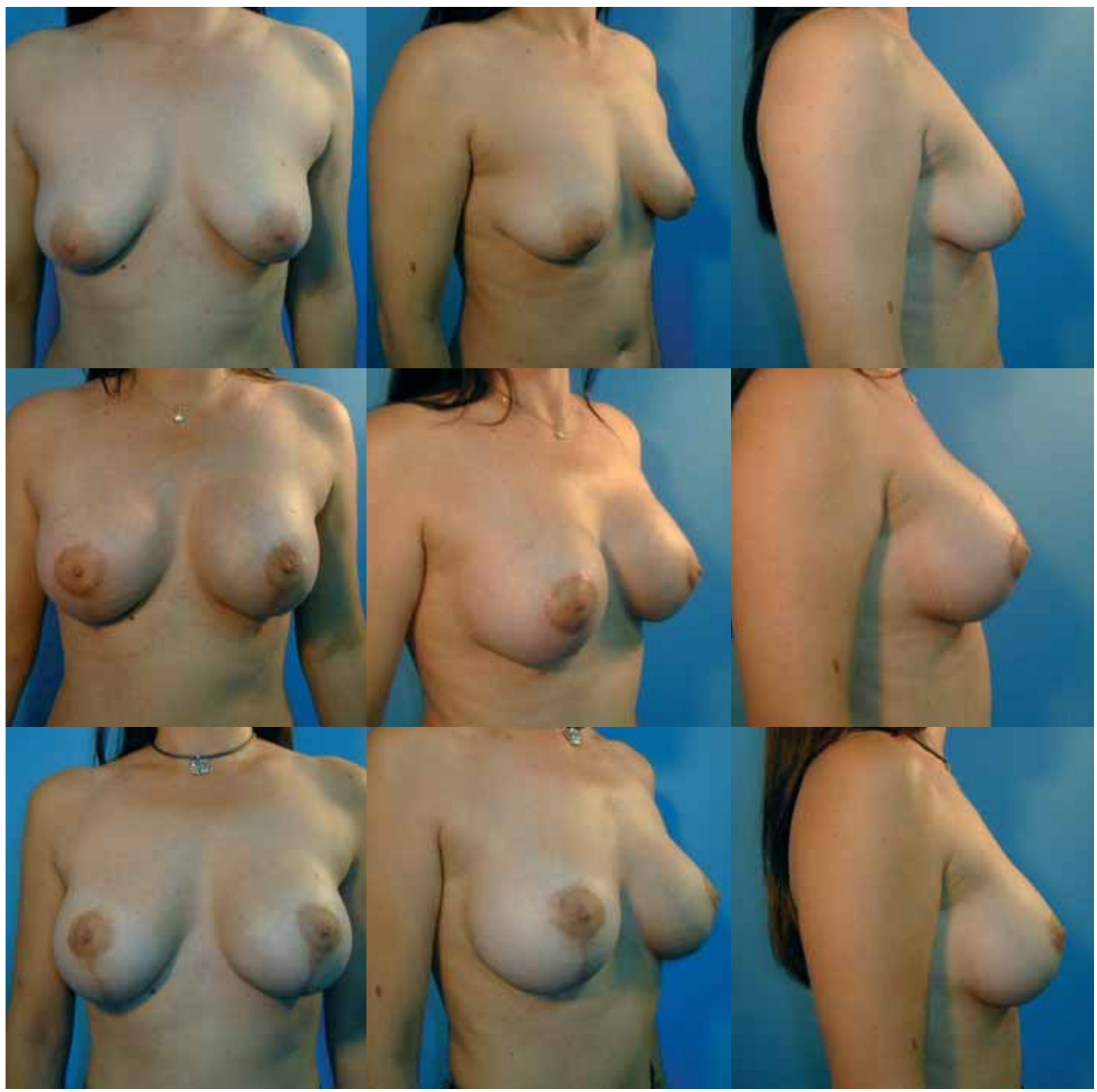

Fig. 12: Paciente 4. Mujer 39 años, Preoperatorio, postoperatorio 1 año y postoperatorio a los 5 años.

\section{Bibliografía}

1. Dufourmentel C, Mouly R. "Chirurgie plastique". In: Pari-Flammarion 1959; Pp 327-370.

2. Lalardrie J, Jouglard JP. "La chirurgie plastique du sein". Paris: masson, 1974;P: 290

3. Regnault P. "Breast ptosis. Definition and treatment". Clin Plast. Surg 1976; 3: 193

4. Bostwick J. "Correctin of the breast ptosis". In: Aesthetic and recent breast surgery. Sant louis: CV Mosby, 1983: Pp209-249.

5. Kirwan L. "Augmentation of the ptotic breast: simultaneous periareolar mastopexy and breast augmentation". Aesth Surg 1999; 19: 34

6. Puckett CL:"Crescent mastopexy and augmentation". Plast. Reconstr Surg. 1985; 75(4): 533.
7. Regnault P. " Breast reduction and mastopexy, an old love story: B Technique update". Aesth. Plast Surg 1990; 14(2): 101

8. Lejour M: "Vertical mammaplasty: early complications after 250 personal consecutive cases". Plast Reconstr Surg. 1999: 104(3):764.

9. Baran CN, Peker F, Ortak T, Sensoz O, Baran NK. "Unsatisfactory results of periareolar mastopexy with or without augmentation and reduction mammaplasty: enlarged areola with flattened nipple". Aesth Plast Surg 2001; 25(4): 286

10. Moreno I. "Mamoplastia de aumento vía axilar". Cir. Plas. Iberolatinoam. 2001. 27 (3): 201

11. Moreno I. "Mastopexia y prótesis". Comunicación. XIV Congreso de la Federación Iberolatinoamericana de Cirugía Plástica.Cancún Méjico 2002.

12. Moreno I, Ribera M. Drever M. "Vertical scar mastopexy with an implant”. Aesth. Plast. Surg. 2003; 27: 406. 


\title{
Comentario al trabajo uMastopexia y prótesis. Revisión a los 5 añosı
}

\author{
Dr. Guillermo Vázquez \\ Director del Capítulo de Mamas \\ de la FILACP \\ Argentina
}

La Ptosis de Mama cuando tiene un buen volumen glandular, puede tratarse con una variedad de técnicas que están descritas en la bibliografía mundial. Pero cuando esta Ptosis está acompañada de hipotrofia glandular, la cirugía se transforma en uno de los desafíos más difíciles que tiene que afrontar el cirujano plástico en la cirugía mamaria. Esto se debe a que por un lado se deberá realizar el tratamiento de la Ptosis con la resección de piel necesaria y por otro lado, restaurar el volumen mamario con un implante. Esta dicotomía que es la de retirar piel y por otro lado aumentar el volumen, es lo que hace que esta cirugía deba ser acompañada por una gran experiencia del cirujano en este tipo de patologías, para poder lograr un buen resultado sin necesidad de retoques posteriores.

Los autores nos presentan su experiencia de 5 años en la cirugía de Mastopexia con inclusión. He leído con mucha atención este trabajo. Sus resultados son muy buenos, pero realmente me surgen muchas dudas sobre su contenido, ya que creo, pueden confundir a los lectores de esta prestigiosa revista.

1. En primer término, los autores, nos informan que han intervenido quirúrgicamente a 150 pacientes. No especifican bajo qué tipo de anestesia fueron intervenidos y no hacen una clasificación de dichos pacientes. No es lo mismo una Ptosis Mamaria donde el Complejo Areola-Pezón (CAP) se encuentra a $3 \mathrm{~cm}$. de su nueva posición o aquella en el que el CAP se encuentra a 8-10 cm., ya que cuánto más sea la distancia que haya que subir el CAP, más larga será la cicatriz vertical y en muchos casos será necesario compensar con una pequeña cicatriz horizontal en el surco submamario, para que la cicatriz vertical no sea visible en el tórax.

2. Con respecto a la colocación del implante en el plano interpectoral (que esa es la terminología correcta, debido a que existen 2 músculos pectorales) y no como habitualmente se lo denomina subpectoral; no sabemos si es submuscular total o parcial ya que no se especifica. Por lo que se aprecia en la fotografía 5, parece ser submuscular total y es sólo una apreciación.

3. Cuando realizan el tallado del colgajo en la fotografía 6, el mismo debería estar dibujado con azul de metileno o cualquier otra sustancia, para que el lector pudiera apreciar la forma del mismo. Los autores dicen que el colgajo es de pedículo interno y superior, pero se visualiza perfectamente en la fotografía, que el mismo, no es a pedículo superior, por lo que crea más confusión en la lectura.

4. La fijación del colgajo en la base del CAP no está explicada. No sabemos si lo fijan con varios puntos o con sutura continua, qué tipo de hilo, a qué nivel del CAP va el punto más superior del colgajo en hora 12 , etc.

5. No especifican en qué plano dejan los drenajes. Teniendo un plano retromuscular y otro retroglandular es importante saber, cuál es el plano elegido para el drenaje. Por lo que se puede apreciar y es sólo una apreciación fotográfica, en la fotografía 5 el drenaje parece estar en posición retromuscular.

6. En los resultados nos hablan de ventajas y desventajas.

Por un lado dicen que es una técnica que deja poca cicatriz (como ventaja) y por otro que deja una cicatriz periareolar y otra vertical (como desventaja). Para cualquier lector esto simplemente es una contradicción.

También afirman que debido al colgajo introducido en la base del CAP se evita la recidiva de la Ptosis, pero no explican cuál sería el mecanismo de acción del colgajo para evitar esta recidiva ya que en un primer momento el colgajo era para la protusión del CAP.

7. Con respecto a las complicaciones no hay ninguna estadística de las mismas. Dicen textualmente: "podríamos citar las propias de la cirugía: infecciones, cicatrices hiperpigmentadas, pero citaremos las que nosotros hemos tenido con la técnica que presentamos..."; por lo que se deduce que no han tenido infecciones, cicatrices hiperpigmentadas, hematomas, dehiscencia de heridas, alteraciones de la sensibilidad, etc.

Los autores nos dan el porcentaje de deflaciones que ellos han sufrido con respecto a los implantes de solución salina, pero obvian hablar de la Contractura Capsular Fibrosa la cual oscila entre 3\% y 10\% según la bibliografía mundial para ese tipo de implantes.

Sí, coincido plenamente con los autores que en la cirugía de Ptosis Mamaria con inclusión debe colocarse el implante en primer término, para luego hacer el tratamiento de la Ptosis. 
Para finalizar es importante recordar que los autores que presentan trabajos para publicar en las revistas científicas de cualquier especialidad, deben tener en cuenta que los mismos deben tener un orden, cumplir con ciertas normativas, ser claros, con toda la información necesaria, ya que el amplio espectro de lectores, puede abarcar desde un médico en formación, hasta un cirujano con amplia experiencia en la especialidad y en el tema.

Cuando un trabajo está titulado como Follow-up, como este, (que traducido al español significa "seguimiento"), debe informar qué cosas los autores han modificado, qué han observado, qué complicaciones han sufrido, etc., en un período determinado con respecto al trabajo original. Esta situación no se manifiesta en lo expuesto por las Dras. Moreno Gallent y Rivera Pons.
La casuística descrita habla de 150 pacientes. Los autores nos muestran sus resultados con fotos de preoperatorio, postoperatorio a 1 año y a 5 años. De los 4 casos presentados, tres están publicados por los mismos autores junto al Dr. Michael Drever en el Aesthetic Plastic Surgery (cita ${ }^{\circ} 12$ de la bibliografía), solamente se le ha agregado el postoperatorio alejado a 5 años.

Las publicaciones de trabajos científicos, deben ser entendidas como una generosa y desinteresada colaboración para la especialidad por parte de los autores, y que la experiencia y la inventiva que poseen, ayude a que en cualquier parte del mundo, algún colega obtenga mejores resultados o evite complicaciones.

\section{Respuesta al comentario del Dr. G. Vázquez}

\section{Dra. I. Moreno Gallent}

Agradezco al Dr. G. Vázquez todos los comentarios que ha efectuado al trabajo que con tanto interés y esfuerzo hemos realizado, dada la dificultad que conlleva conseguir la mejoría y la corrección en los casos de hipotrofia mamaria con ptosis.

Hemos considerado las múltiples apreciaciones y dudas que nos dice que le surgen y vamos a intentar contestar a algunas de ellas, puesto que el trabajo supone un seguimiento ("follow-up") de la técnica que venimos realizando (con una experiencia de 20 años) y que fue publicada en el año 2003 en la revista Aesthetic Plastic Surgery.

Todos los pacientes se intervienen bajo anestesia general y en ambiente hospitalario. Al tratarse de una hipotrofia mamaria la distancia del CAP nunca va a sobrepasar los $4 \mathrm{~cm}$. La edad de las pacientes osciló entre 21 años y 65 años de edad. Describimos el plano subpectoral o retropectoral; hasta ahora en la literatura suele utilizarse la nomenclatura de planos de colocación de los implantes: subglandular, retropectoral o subpectoral y subfascial. Dado que los colgajos que utilizamos son pequeños y la propia sutura entre los pilares de la glándula los mantiene en su sitio, utilizamos materiales reabsorbibles tales como Safil o PDS. Siempre colocamos los drenajes en posición subpec- toral, al principio dejábamos 4 drenajes ( 2 en posición subglandular y otros 2 en posición subpectoral), pero la propia experiencia ha hecho que los dejemos en el plano subpectoral.

En general esta técnica solo nos aporta ventajas como citamos en el trabajo, por lo que no hemos realizado cambios con el tiempo, sí en función de los pacientes, no hay dos paciente con el mismo grado de hipotrofia ni de ptosis. Las bases de la misma técnica no han cambiado. Hemos mostrado las fotos de las pacientes que consideramos la muestra de la técnica tras cinco años porque pensamos que ese era el objetivo del trabajo, el "follow-up" de la técnica.

Hemos intentado con este trabajo dar a conocer la manera que tenemos de resolver la complicada situación de las pacientes que presentan este problema y aportar una vía más de resolución. Sentimos no haber satisfecho sus gustos con esta técnica. Sin duda y aprovechando la realización del próximo Congreso Nacional de la Sociedad Española en nuestra capital, Valencia, en 2007, le invitamos a que vea nuestro trabajo y aclararle todas las dudas que presente.

Le agradezco sinceramente todos los comentarios y acepto sus discrepancias basadas en los argumentos sólidos que el rigor científico merece. 